International Journal of Poultry Science 3 (7): 438-441, 2004

(C) Asian Network for Scientific Information, 2004

\title{
An Epizootic of Combined Clostridium perfringens, Eimeria Spp. and Capillaria Spp. Enteritis and Histomonas Spp. Hepatitis with Escherichia coli Septicemia in Bobwhite Quails (Colinus virginianus)
}

\author{
Zaher Ahmad Radi \\ Tifton Diagnostic and Investigational Laboratory, College of Veterinary Medicine, \\ The University of Georgia, P.O. Box 1389, Tifton, Georgia 31793, USA \\ E-mail: zradi@tifton.uga.edu
}

\begin{abstract}
Four young Bobwhite quails (Colinus virginianus) were submitted to the Veterinary Diagnostic and Investigational Laboratory of The University of Georgia for necropsy. The quails were from a flock which had a history of anorexia, weakness, diarrhea, dehydration, weight loss and acute death. The total number of mortalities within two weeks was 80 out of approximately 900 birds. Multi focal sub-serosal intestinal ulcerations with subsequent perforation and peritonitis and Multi focal necrotizing hepatitis were noted on gross examination. Microscopic examination of the small and large intestine revealed severe necroulcerative and heterophilic enterocolitis and typhlitis with bacterial colonization and intralesional Capillaria spp. Fecal examination revealed moderate amounts of Capillaria spp. and few Eimeria spp. Clostridium perfringens was isolated from the intestine. In addition, there was Multi focal necrogranulomatous hepatitis with intralesional protozoal trophozoites consistent with Histomonas spp. morphology. Escherichia coli was isolated from specimens of liver, spleen and intestine.
\end{abstract}

Key words: Bobwhite quails, Capillaria, C. perfringens, Eimeria, Histomonas, E. coli

\section{Introduction}

Clostridial enteritis is a relatively common disease in avian species. Clostridium perfringens is a large Gramnegative, spore-forming, anaerobic bacterium that causes necrotic enteritis in chickens and turkeys (Broussard et al., 1986; Droual et al., 1994). In quails, outbreaks of the highly contagious ulcerative enteritis caused by Clostridium colinum have been described (Berkhoff and Kanitz, 1976). Fecal-oral infection usually occurs in clostridial enteritis.

Intestinal coccidiosis (Eimeria spp.) is a common protozoal disease in various avian species and Eimeria spp. are highly host specific (Jordan, 1990). Various species of Eimeria have been isolated from quails (Duszynski and Gutierrez, 1981). Coccidia produce lesions in the intestinal tract by destruction of epithelial and submucosal cells in which they develop and multiply (Jordan, 1990). Oocysts are easily transported to poultry farms by boots, shoes, clothing, and vehicle wheels. Also, people are important vectors of the coccidia (Jordan, 1990).

Capillaria spp. are nematodes that parasitize the intestinal mucosa in many avian species. These thin and long nematodes are not host specific and may have a direct or indirect life cycle. They usually live in the small intestinal mucosa and some in the esophagus, crop, and cecum (Jordan, 1990). Capillaria spp. infection is infrequently found in Bobwhite quails (Davidson et al., 1991).

Histomonas meleagridis is a protozoan parasite that has been reported in turkeys, chickens, peafowl, partridges and grouse (Lund and Chute, 1972; Jordan, 1990). Pheasants and quails are considered to be vectors of $H$. meleagridis and relatively resistant to infection (Lund and Chute, 1972; Zeakes et al., 1981). However, a natural outbreak of atypical histomoniasis has been reported in quails (Dhillon et al., 1980).

Septicemic colibacillosis caused by Escherichia coli is an infectious avian disease that has been commonly reported in chicken, turkeys, ducks, and quails (da Silva et al., 1989). E. coli is linked to a wide range of clinical diseases, such as septicemia, polyserositis, coligranuloma, enteritis, yolk sac infection, airsacculitis, peritonitis, cellulites and omphalitis (Dho-Moulin and Fairbrother, 1999). Diseases associated with E. coli cause great economic losses in avian industries. The purpose of this paper is to describe a combined $C$. perfringens, Capillaria spp., and Eimeria spp. enteritis, and Histomonas spp. hepatitis with $E$. coli septicemia in a flock of Bobwhite quails (Colinus virginianus).

\section{Materials and Methods}

Case history: On May 15 2003, four dead young Bobwhite quails (Colinus virginianus) were submitted to the Veterinary Diagnostic and Investigational Laboratory, The University of Georgia, Tifton, Georgia for necropsy. The owner stated that the birds were weak, anorexic, had diarrhea, weight loss and had died within 1 day of showing clinical signs. The mortality began on May 1. The total mortalities within two weeks were 80 out of 
Zaher Ahmad Radi: Bobwhite quails enteritis, hepatitis, and septicemia

approximately 900 birds. No treatment was given to the birds.

Pathology: The birds were examined externally, complete necropsy was done and gross lesions were recorded. Tissue samples from different organs including lung, heart, liver, kidney, crop, proventriculus, gizzard, intestine and brain were collected and fixed in $10 \%$ neutral buffered formalin, processed and embedded in paraffin blocks. Sections were cut at 4-5 $\mu \mathrm{m}$, mounted on glass slides, and stained with haematoxylin and eosin.

Bacteriology: Liver, spleen and intestine tissue were cultured aerobically at $37^{\circ} \mathrm{C}$ on $5 \%$ bovine blood agar plates (Sparks, MD, USA). Intestines were cultured aerobically for Salmonella species on Hektoen Enteric (HE) Agar Plates (Sparks, MD, USA) and in Tetrathionate Broth (Remel, KS, USA). The HE plates and the Tetrathionate broth cultures were incubated aerobically at $37^{\circ} \mathrm{C}$ for 24 hours and subcultures made from the Tetrathionate broth onto new HE plates and were incubated similarly. Both sets of $\mathrm{HE}$ plates were examined for suspect Salmonella colonies.

Intestines were cultured anaerobically on WilkinsChalgren Agar with 5\% bovine blood (Sparks, MD, USA) and on Phenyl Ethyl Alcohol Agar with 5\% bovine blood (Sparks, MD, USA) in a Forma Scientific Anaerobic System at $35^{\circ} \mathrm{C}$. They were observed for growth of anaerobic organisms every 24 hours for 5 days.

Significant aerobic organisms were separated to obtain pure cultures and then run on conventional media or set up on the Trek Sensititre identification and sensitivity system using the GNID, GPID and CMV1BAVF panels. Anaerobic isolates were sub-cultured onto Tryptic Soy Blood Agar with 5\% bovine blood and set up on the RapANID System (Remel, KS, USA) for identification.

Electron microscopy: Intestinal contents were examined for viruses by electron microscopy of negatively stained samples.

Parasitology: Intestinal contents were examined for parasites by direct fecal examination and fecal flotation. They were also examined by modified acid fast stain for Crypto sporidium.

\section{Results}

All the birds submitted were in very poor body condition with very prominent keel and breast musculature was severely atrophied. They were severely dehydrated and emaciated. A moderate amount of fecal material was pasted to the feathers surrounding the vent. Multi focal sub-serosal intestinal ulcerations were observed in all birds. Some birds had subsequent perforation of these ulcers and peritonitis was noticed on gross examination (Fig. 1). The spleens were moderately enlarged (Fig. 1). The small and large intestinal lumen contained foulsmelling material and was lined by a tan, friable diphtheritic membrane. The livers were moderately enlarged, friable, and pale tan. Multi focally scattered throughout the livers were round to irregular, circumscribed, flat to sunken white-tan foci ranging in size from 0.5 to $2 \mathrm{~cm}$ (Fig. 3). There were no other significant lesions.

Histologically, the intestines had marked diffuse ulceration and extensive loss of mucosal lining epithelium and lamina propria with replacement by large amounts of eosinophilic cellular and karyorrhectic debris, extravasated erythrocytes (hemorrhage), edema fluid and fibrin exudation. There were large numbers of degenerate heterophils, scattered multinucleated giant cells, lymphocytes and macrophages admixed with the fibrinonecrotic debris, forming a diphtheritic membrane (Fig. 2). Multi focally within the necrotic cecal lamina propria and the submucosa, there were many protozoal trophozoites consistent with the morphology of Histomonas spp. Multiple sections of nematodes (Capillaria spp.) were present within the lumen (Fig. 2). Direct fecal examination and fecal flotation demonstrated moderate amounts of Capillaria spp. and few Eimeria spp.

The liver had Multi focal to coalescing areas of necrosis and inflammation with loss of hepatocytes. In these areas there were abundant cellular and karyorrhectic debris, large infiltrates of macrophages and lymphocytes, and scattered foreign body-type multinucleate giant cells, and heterophils (Fig. 4). Multi focally there were many round, eosinophilic to amphophilic protozoal trophozoites that are often surrounded by a clear zone consistent with the morphology of Histomonas spp. (Fig. 4). These organisms were periodic-acid-Schiff-positive and were present singly or in clusters (Fig. 5). The kidneys had minimal Multi focal tubular dilation and degeneration. No other significant lesions were noted.

E. coli was isolated from specimens of liver, spleen, and intestine. Clostridium perfringens was isolated from the intestine. Direct fecal examination and fecal flotation revealed a light load of Eimeria spp. and moderate load of Capillaria spp. Modified acid fast stain for Crypto sporidium was negative. Fecal material examined by negative-stain electron microscopy was negative for viruses.

\section{Discussion}

Gross and histological lesions of enteritis and hepatitis explain the severe emaciation, dehydration and weight loss in these birds. Clostridial enteritis is a common problem in avian species (Ficken and Wage, 1997). Both Clostridium perfringens and Clostridium colinum have been documented as causes of avian ulcerative 
Zaher Ahmad Radi: Bobwhite quails enteritis, hepatitis, and septicemia

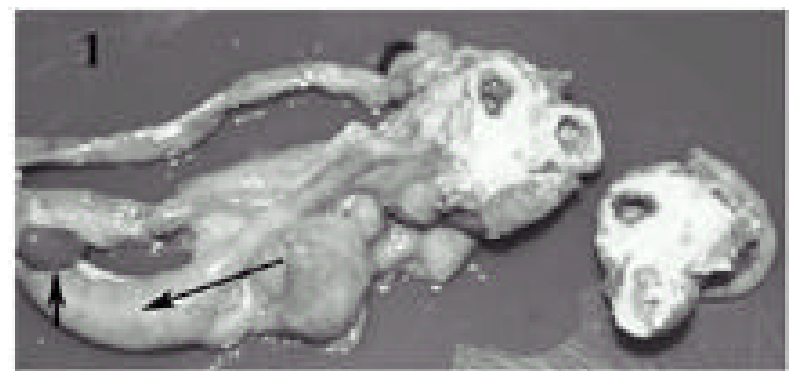

Fig. 1: Intestine with Multi focal sub-serosal ulcerations (long arrow), perforation and peritonitis and enlarged spleen (short arrow) in an affected quail

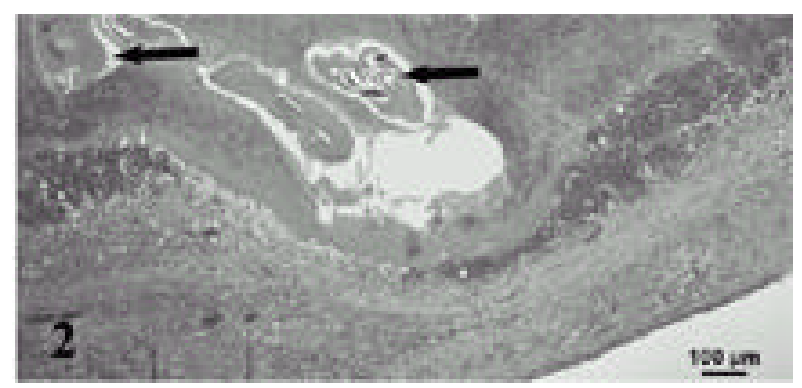

Fig. 2: Photomicrograph of the intestine with marked diffuse ulceration, necrosis, and inflammation with multiple cross sections of nematodes (Capillaria spp.) (arrows) (HE)

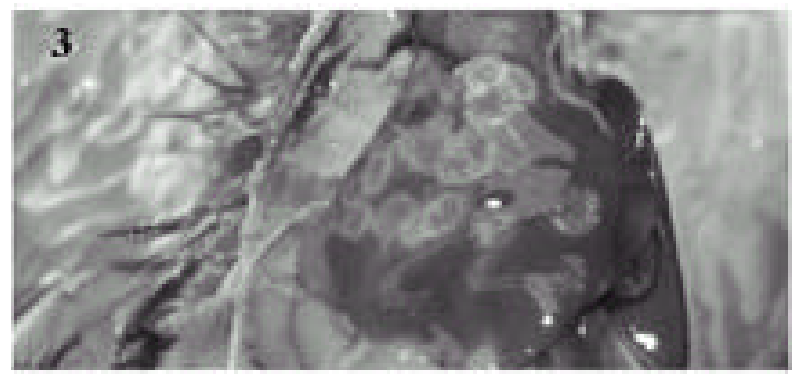

Fig. 3: Liver with multiple white-tan foci of necrosis infected with Histomonas spp. in a quail

enteritis. The microscopic lesions together with the isolation of $C$. perfringens in this case are similar to previously reported avian clostridial enteritis (Ficken and Wage, 1997). To our knowledge, C. perfringens enteritis has not been reported in quails. Damage to the intestinal mucosa is a predisposing factor for clostridial infection (Ficken and Wage, 1997). It has been reported that coccidiosis and stress are predisposing factors for ulcerative and necrotic enteritis (Davis, 1973; Al-Sheikhly and Al-Saieg, 1980). Poor hygiene and management are also predisposing factors to Clostridial infection (Ficken and Wage, 1997). Contaminated feed and litter with $C$.

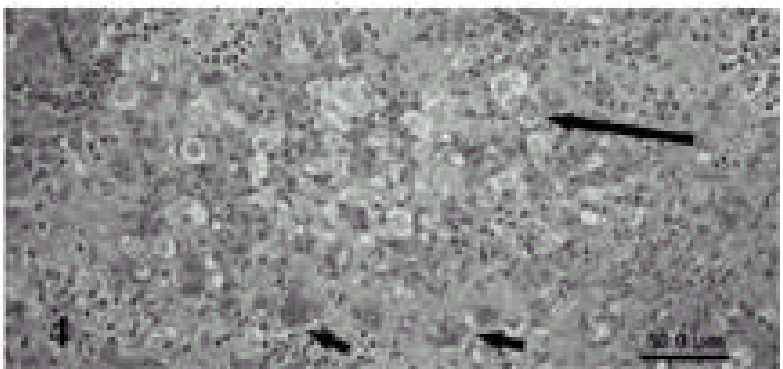

Fig. 4: Photomicrograph of the liver with Multi focal to coalescing necrotizing and granulomatous inflammation. Note Histomonad organisms (long arrows) and multinucleated giant cells (short arrows) (HE)

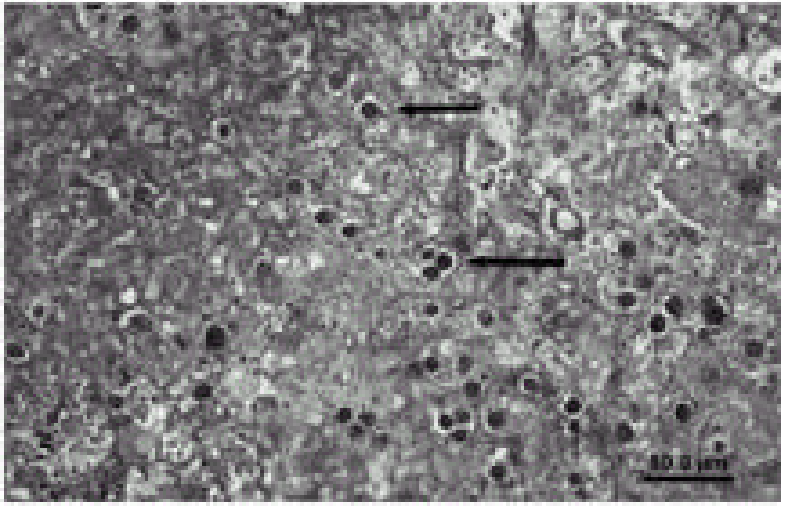

Fig. 5: Histomonad organisms (arrows) in an affected quail liver (periodic-acid-Schiff-positive)

perfringens have been incriminated as sources of infection (Ficken and Wage, 1997). In this case, the combined intestinal mucosal injury and trauma due to Eimeria spp. and Capillaria spp. infection, poor hygiene and management, and increased clostridial proliferation with subsequent feed and litter contamination have predisposed the birds in this case to increased susceptibility to clostridial infection.

Histomoniasis (blackhead disease) is a disease caused by the protozoan parasite Histomonas meleagridis (McDougald, 1997). Histomonas meleagridis is transmitted horizontally through intermediate hosts such as intestinal nematodes Heterakis gallinae and the earthworm. Bobwhite quails (Colinus virginianus) are usually considered a reservoir for the disease (Kellogg and Reid, 1970). The severity of histomoniasis is increased with bacterial co-infection with C. perfringens and E. coli (MaDougald, 1997). Although a natural outbreak of atypical histomoniasis has been reported in quails (Dhillon et al., 1980), no concurrent bacterial and protozoal infection has been reported. The present article demonstrates a concurrent bacterial and protozoal infection. 
Zaher Ahmad Radi: Bobwhite quails enteritis, hepatitis, and septicemia

E. coli infection in avian species can cause many clinical manifestations (Barnes et al., 1997). Colisepticemia is one of the avian disease manifestations of $E$. coli infection and has been reported in chickens, turkeys and quails (Barnes et al., 1997; Arenas et al., 1999). In our case, E. coli was isolated from specimens of liver, spleen, and intestine.

To the author's knowledge, this is the first report of combined $C$. perfringens, Eimeria spp. and Capillaria spp. enteritis and Histomonas spp. hepatitis with E. coli septicemia in quails. Avian pathologists should consider multiple etiologies for enteritis in quails.

\section{Acknowledgments}

The author would like to thank the VDLI staff for their technical help.

\section{References}

Al-Sheikhly, F. and A. Al-Saieg, 1980. Role of Coccidia in the occurrence of necrotic enteritis of chickens. Avian Dis., 24: 324-333.

Arenas, A., S. Vicente, I. Luque, J.C. GomezVillamandos, R. Astorga, A. Maldonado and C. Tarradas, 1999. Outbreak of septicaemic colibacillosis in Japanese quail (Coturnix coturnix japonica). Zentralbl. Veterinarmed. B, 46: 399-404.

Barnes, H.J. and W.B. Gross, 1997. Colibacillosis. In: Calnek, B.W. Barens, H.J., Beard, C.W. McDougald, L.R. and Saif, Y.M. (EDs.), Diseases of Poultry, $10^{\text {th }}$ ed. lowa State University Press, Ames, lowa, pp: 131141.

Berkhoff, G.A. and C.L. Kanitz, 1976. Fluorescent antibody test in diagnosis of ulcerative enteritis. Avian Dis., 20: 525-533.

Broussard, C.T., C.L. Hofacre, R.K. Page and O.J. Fletcher, 1986. Necrotic enteritis in cage-reared commercial layer pullets. Avian Dis., 30: 617-619.

Davis, R.B., 1973. Ulcerative enteritis in chickens: coccidiosis and stress as predisposing factors. Poult. Sci., 52: 1283-1287.

da Silva, P.L., H.E. Coelho, S.C. Ribeiro and P.R. Oliveira, 1989. Occurrence of coligranulomatosis in coturnix quail in Uberlandia, Minas Gerais, Brazil. Avian Dis., 33: 590-593.
Davidson, W.R., F.E. Kellogg, G.L. Doster and C.T. Moore, 1991. Ecology of helminth parasitism in bobwhites from northern Florida. J. Wildl. Dis., 27: 185-205.

Dho-Moulin, M. and J.M. Fairbrother, 1999. Avian pathogenic Escherichia coli (APEC). Vet. Res., 30: 299-316.

Dhillon, A.S., R.W. Winterfield, H.L. Thacker, K.R. Kazacos and L.J. Alby, 1980. Atypical histomoniasis in Bobwhite quail. Avian Dis., 24: 510-516.

Droual, R., H.L. Shivaprasad and R.P. Chin, 1994. Coccidiosis and necrotic enteritis in turkeys. Avian Dis., 38: 177-183.

Duszynski, D.W. and R.J. Gutierrez, 1981. The coccidia of quail in the United States. J. Wildl. Dis., 17: 371379.

Ficken, M.D. and D.P. Wage, 1997. Necrotic enteritis. In: Calnek, B.W. Barens, H.J., Beard, C.W. McDougald, L.R. and Saif, Y.M. (EDs.), Diseases of Poultry, $10^{\text {th }}$ ed. lowa State University Press, Ames, lowa, pp: 261264.

Jordan, F.T.W., 1990. Parasitic Diseases. In: F.T.W. Jordan, (EDs.), Poultry Diseases, $3^{\text {rd }}$ ed. Cambridge University Press, Cambridge, UK, pp: 226-250.

Kellogg, F.E. and W.M. Reid, 1970. Bobwhites as possible reservoir hosts for blackhead in wild turkeys. J. Wildl. Manage., 34: 155-159.

Lund, E.E. and A.M. Chute, 1972. Reciprocal responses of eight species of galliform birds and three parasites: Heterakis gallinarum, Histomonas meleagridis, and Parahistomonas wenrichi. J. Parasitol., 58: 940-945.

McDougald, L.R., 1997. Other protozoan disease of the intestinal tract. In: Calnek, B.W. Barens, H.J., Beard, C.W. McDougald, L.R. and Saif, Y.M. (EDs.), Diseases of Poultry, $10^{\text {th }}$ ed. Iowa State University Press, Ames, lowa, pp: 890-899.

Zeakes, S.J., H.F. Hansen and R.J. Robel, 1981. Increased susceptibility of bobwhites (Colinus virginianus) to Histomonas meleagridis after exposure. Avian Dis., 25: 981-987. 